\title{
Análisis espacial de mortalidad infantil y servicios de salud en municipios indígenas de Yucatán, México, 1990-2000.
}

\author{
Artículo Original
}

Rosa M. Méndez-González, María D. Cervera-Montejano.

Departamento de Ecología Humana, Centro de Investigación y de Estudios Avanzados del I.P.N. Unidad Mérida, Mérida, Yucatán, México.

\section{RESUMEN.}

Objetivo. Conocer, desde la perspectiva de la geografía de la salud, la distribución espacial de mortalidad infantil y servicios de salud en municipios indígenas (MI) de Yucatán, México, de 1990 a 2000, analizando las diferencias espaciotemporales para detectar zonas que requieren atención prioritaria.

Material y métodos. La unidad de análisis fue el municipio. Se consideró MI cuando más del $40 \%$ de su población mayor de 5 años era hablante de lengua indígena. Se estimaron las tasas de mortalidad infantil (TMI) de los MI, se estratificaron en tres grados para facilitar la visualización de la distribución territorial (alto, medio y bajo). Para los servicios de salud se utilizó la presencia de unidades médicas según nivel de atención $\left(1^{\circ}, 2^{\circ}\right.$ y $\left.3^{\circ}\right)$ y número de médicos $/ 1,000$ habitantes. Se realizaron análisis cartográficos simples y relacionales, empleando un Sistema de Información Geográfica (SIG).

Resultados. De 1990 a 2000 disminuyeron los
MI. Las TMI descendieron, pero la proporción de MI con grado alto de TMI aumentó. En la mayoría de los MI predominaron las unidades médicas de primer nivel; en 2000, disminuyeron las unidades de segundo nivel en los MI. En ambos años prevalecieron los MI que tenían menos de un médico/1,000 habitantes. La proporción de MI que contaban con unidades médicas de primer nivel y tenían grado alto de TMI aumentó de 1990 a 2000, también los que tuvieron grado alto de TMI y menos de un médico/1,000 habitantes.

Comentarios. El SIG permitió visualizar y analizar en espacio y tiempo las variables e identificar sus relaciones. Así, fue posible ubicar que las zonas sur y oriente fueron las más desfavorecidas.

(Rev Biomed 2006; 17:108-117)

Palabras clave: municipios indígenas; mortalidad infantil; servicios de salud; cambios 1990-2000.

\section{SUMMARY.}

Spatial analysis of infant mortality and health

Solicitud de sobretiros: Rosa M. Méndez-González. Departamento de Ecología Humana, CINVESTAV. IPN. Unidad Mérida, Km. 6 Antigua Carretera a Progreso, C.P. 97310, Mérida, Yucatán, México.

Tel. 124-21-11 Fax 981-46-70 Correo electrónico: rmmg@mda.cinvestav.mx

Recibido el 14/Noviembre/2005. Aceptado para publicación el 12/Junio/2006.

Este artículo está disponible en http://www.uady.mx/sitios/biomedic/revbiomed/pdf/rb061724.pdf

Vol. 17/No. 2/Abril-Junio, 2006 
RM Méndez-González, MD Cervera-Montejano.

services in indigenous municipalities in the state of Yucatan, Mexico from 1990 - 2000.

Objective. To determine, from the perspective of the geography of the health, the spatial distribution of infant mortality and health services in indigenous municipalities (IM) in the state of Yucatan, Mexico, from 1990 to 2000, analyzing the space-temporal differences to detect zones that require high-priority attention.

Material and methods. The analysis unit was the municipality. IM was defined when more than $40 \%$ of the population five years or older, spoke an indigenous language. The Infant Mortality Rates (IMR) were calculated for each IM and stratified into three levels to facilitate the visualization of the territorial distribution (high, average and low). For the health services, presence of medical units were used according to the level of attention $\left(1^{\circ}, 2^{\circ}\right.$ and $\left.3^{\circ}\right)$, also the relation of number of physician/1,000 inhabitants was used. First, we applied cartographic analysis to each variable; second, we used it to relate variables the each other. Geographical Information System (GIS) was the tool for both cases.

Results. From 1990 to 2000, the number of indigenous municipalities decreased. IMR descended, but the proportion of IM with a high degree of IMR increased. In most IM first level medical units predominated; in 2000, the second level units in IM diminished. In both years, the majority of municipalities had less than one physician/1,000 inhabitants. The proportion of IM that counted on first level medical units and had a high degree of IMR increased from 1990 to 2000, as well as also those that had a high degree of IMR and less than one physician/1,000 inhabitants.

Commentaries. The GIS allowed to visualize and to analyze the variables in both space and time and to identify their relationship. Thus, it was possible to locate the southerm and eastern zones of Yucatan as the most deteriorated areas of the state.

(Rev Biomed 2006; 17:108-117)

Key words: indigenous municipalities; infant mortality; heath services; changes 1990-2000.

\section{INTRODUCCIÓN.}

Las diferencias observadas en los perfiles epidemiológicos se relacionan con la desigualdad en el crecimiento y distribución del ingreso (1). En particular, la Organización Panamericana de la Salud (OPS) y la Organización Mundial de la Salud (OMS) han destacado su preocupación por la mortalidad excesiva que se presenta en las poblaciones indígenas debida a causas prevenibles asociadas a la marginación $(2,3)$.

México es el país con mayor proporción de población indígena en América Latina, en donde representa el 11\% de la población total. La mayoría de los indígenas vive en áreas rurales, en los estados del sur y sureste, en condiciones de alta marginación (4). Los principales indicadores de salud muestran que en esta población, la esperanza de vida al nacer es de 69 años contra 74 de la población nacional. El riesgo de morir de una mujer indígena durante el embarazo, parto o puerperio es casi tres veces más alto que el de una mujer no indígena. La prevalencia de desnutrición crónica es de $44 \%$ mientras que a nivel nacional es de $17.7 \%$. La probabilidad de morir durante los primeros cinco años de vida en los municipios con mayor proporción de población indígena es de 53.1/1,000 nacidos vivos contra 25/1,000 nacidos vivos en municipios con menor proporción, y la tasa de mortalidad infantil es dos veces superior a la nacional $(1,5,6)$.

La mortalidad infantil es uno de los indicadores más sensibles de la desigualdad social y de los cambios que se producen en las sociedades, se relaciona con la disponibilidad y el acceso a los servicios básicos de salud y sus principales causas -afecciones originadas en el período perinatal, las enfermedades infecciosas intestinales y neumonías- pueden prevenirse fácilmente (7-13). En los últimos años se ha incrementado en México la infraestructura de salud en las localidades indígenas: en 1990 había 537 unidades médicas de primer nivel y cuatro de

\section{Revista Biomédica}




\section{Mortalidad infantil y servicios de salud en Yucatán, México, 1990-2000.}

segundo nivel y en 2000, aumentaron a 2,095 y 31, respectivamente (6). Sin embargo, aún existen deficiencias y desigualdades en la distribución de los recursos materiales y humanos. Este es el caso de la relación médicos por habitantes. Por ejemplo, de acuerdo con el criterio de la Organización Panamericana de la Salud (12), que establece una relación aceptable cuando llega a un médico/1,000 habitantes, para el país es apenas aceptable con 1.38/1,000, mientras que tanto en las zonas indígenas de Oaxaca como en el estado en su conjunto es deficiente ya que es de 0.13 en las primeras y de 0.94 en el segundo (6). A estos problemas se agregan las barreras geográficas, sociales, económicas y culturales que interfieren con la accesibilidad de las poblaciones indígenas a los servicios de salud y la integración de los recursos humanos locales y, por tanto, limitan la posibilidad de recibir atención preventiva y curativa para evitar la mortalidad infantil.

Considerando que Yucatán es el estado con mayor proporción de población hablante de lengua indígena (14) se realizó un estudio de geografía de la salud para conocer la distribución espacial de la mortalidad infantil, las unidades médicas y los médicos por 1,000 habitantes en municipios indígenas, así como los cambios ocurridos de 1990 a 2000. Mediante un Sistema de Información Geográfica se analizaron las diferencias espaciotemporales y se identificaron los municipios indígenas que requieren de una atención prioritaria por presentar tasas altas de mortalidad infantil y deficiencia en los servicios de salud.

\section{MATERIAL Y MÉTODOS.}

La unidad de análisis fue el municipio y se manejó como patrón de área cartográfica para estudiar la distribución en espacio y tiempo de las variables, divididas en clases o categorías.

a) Población indígena. Con base en la información de los XI y XII Censos Generales de Población y Vivienda del INEGI $(15,16)$, se clasificó al municipio como indígena o no indígena, siguiendo el criterio de la SSA-INI
(17), que considera como indígenas a aquellos donde más del $40 \%$ de su población de 5 años y más habla lengua indígena.

b) Mortalidad infantil. Se estimaron las tasas de mortalidad infantil (TMI) de todos los municipios de Yucatán. Se obtuvo información sobre nacimientos y defunciones de menores de un año de edad, por lugar de residencia de 1990 a 2000 del Sistema Municipal de Bases de Datos (SIMBAD) del INEGI (18). Las TMI se calcularon de la forma estándar. Debido a las variaciones que normalmente se observan en las tasas anuales de mortalidad y a que un solo valor no refleja el comportamiento de la mortalidad en una población, en lugar de considerar solamente la TMI del año 1990 y la TMI del 2000, se optó por estimar dos valores promedio para cada municipio. El primero corresponde al promedio de las TMI del período 1990-1994 y que originó las TMI 1990; y el segundo al promedio de las TMI 1995-2000, que produjo las TMI 2000.

Una vez que se determinó que las TMI tenían una distribución cercana a la normal, se estratificaron utilizando el método percentilar (quintiles). Este fue seleccionado siguiendo la recomendación de su empleo en el área de geografía de la salud para obtener clases consistentes, especialmente cuando se comparan mapas en diferentes períodos (19). Los datos se procesaron con el SPSS versión 8.0 y los resultados mostraron los valores mínimo y máximo de las TMI así como los puntos de corte de cada intervalo, dando lugar a cinco categorías o grados de mortalidad infantil: muy alto, alto, medio, bajo y muy bajo.

Posteriormente, considerando que para llevar a cabo los análisis espaciales se recomienda utilizar pocas categorías a fin de facilitar la visualización de su distribución territorial, se redujeron de cinco a tres los grados de mortalidad infantil. Los tres grados resultantes fueron: alto, en el que se juntaron los grados muy alto y alto; el grado medio permaneció sin cambios y en el grado bajo se agruparon los grados bajo y muy bajo. No se encontraron diferencias estadísticamente 


\section{RM Méndez-González, MD Cervera-Montejano.}

significativas entre los valores de los intervalos de los cinco y los tres grados $\left(X^{2}=1.74, p=0.40\right)$. La estratificación de las tasas de mortalidad infantil para 1990 y 2000 se presenta en el cuadro 1.

c) Servicios y recursos para la atención de la salud. Se utilizaron la presencia de unidades médicas según nivel de atención $\left(1^{\circ}, 2^{\circ}\right.$ y $\left.3^{\circ}\right)$ y la relación médicos/1,000 habitantes de 1990 y 2000 como indicadores. La información se obtuvo de los Anuarios Estadísticos de Yucatán $(20,21)^{*}$. El número de médicos/1,000 habitantes se clasificó en las siguientes categorías: 0) no se registraron médicos; 1) menos de 1 médico/1,000 habitantes; 2) de 1 a 2 médicos/1,000 habitantes; 3) más de 2 médicos/1,000 habitantes. Se consideró deficiencia de servicio médico cuando el número de médicos/1,000 habitantes era menor de 1, de acuerdo a la propuesta de la OPS (12).

Análisis y procesamiento de la información.

Al tratarse de un estudio de geografía de la salud, se utilizó el análisis espacial simple y relacional basado en la cartografía para identificar relaciones entre mortalidad infantil y servicios y recursos para la atención de la salud en municipios indígenas en 1990 y 2000 y se comparó la distribución de estas relaciones entre ambos años para identificar cambios.
Como primer paso se realizó un análisis cartográfico simple, es decir se elaboraron mapas de cada variable para cada año por separado, para identificar sus patrones de distribución. Posteriormente, se efectuaron análisis relacionales de los dos años entre municipios indígenas que contaban con unidades médicas de primer nivel y presentaron grado alto de mortalidad infantil; y de municipios indígenas que tuvieron grado alto de mortalidad infantil y menos de un médico/1,000 habitantes. Para los análisis espacio-temporales se construyó un Sistema de Información Geográfica (SIG) empleando el ARC/VIEW 3.2.

\section{RESULTADOS.}

\section{Análisis cartográficos simples.}

Por limitaciones de espacio, no se incluyó la representación cartográfica de los análisis simples. Entre 1990 y 2000, el número de municipios indígenas disminuyó, pasando de $82 \%$ a $72 \%$. La disminución ocurrió en municipios aledaños a los costeros. Es probable que el descenso haya ocurrido por pérdida del maya como lengua materna o por migración de la población indígena.

Entre 1990 y 2000, las tasas de mortalidad infantil descendieron en Yucatán, pasando de 24.07/ 1,000 nacidos vivos registrados (nvr) a 13.83/1,000 nvr. En general, durante ese período se mantuvieron

\section{Cuadro 1}

Estratificación de las tasas de mortalidad infantil 1990 y 2000

Yucatán, México.

\begin{tabular}{lcccc}
\hline & \multicolumn{2}{c}{1990} & \multicolumn{2}{c}{2000} \\
Grados & Límite del intervalo & \multicolumn{2}{c}{ Límite del intervalo } \\
(TMI/1,000 nvr) & Inferior & Superior & 5.59 & 16.52 \\
\hline 1 (Bajo) & 10.92 & 18.43 & 16.60 & 19.48 \\
2 (Medio) & 18.61 & 21.54 & 19.77 & 38.23 \\
3 (Alto) & 21.57 & 63.70 & & Superior \\
\hline
\end{tabular}

Fuente: INEGI (Sistema Municipal de Bases de Datos), 2003

*Municipio indígena: aquel en el que más del 40\% de su población de 5 años y más habla lengua indígena (SSA-INI, 1992)

*No se incluyó el sector privado por no encontrarse la información desagregada a nivel municipal para ambos años.

\section{Revista Biomédica}


Mortalidad infantil y servicios de salud en Yucatán, México, 1990-2000.

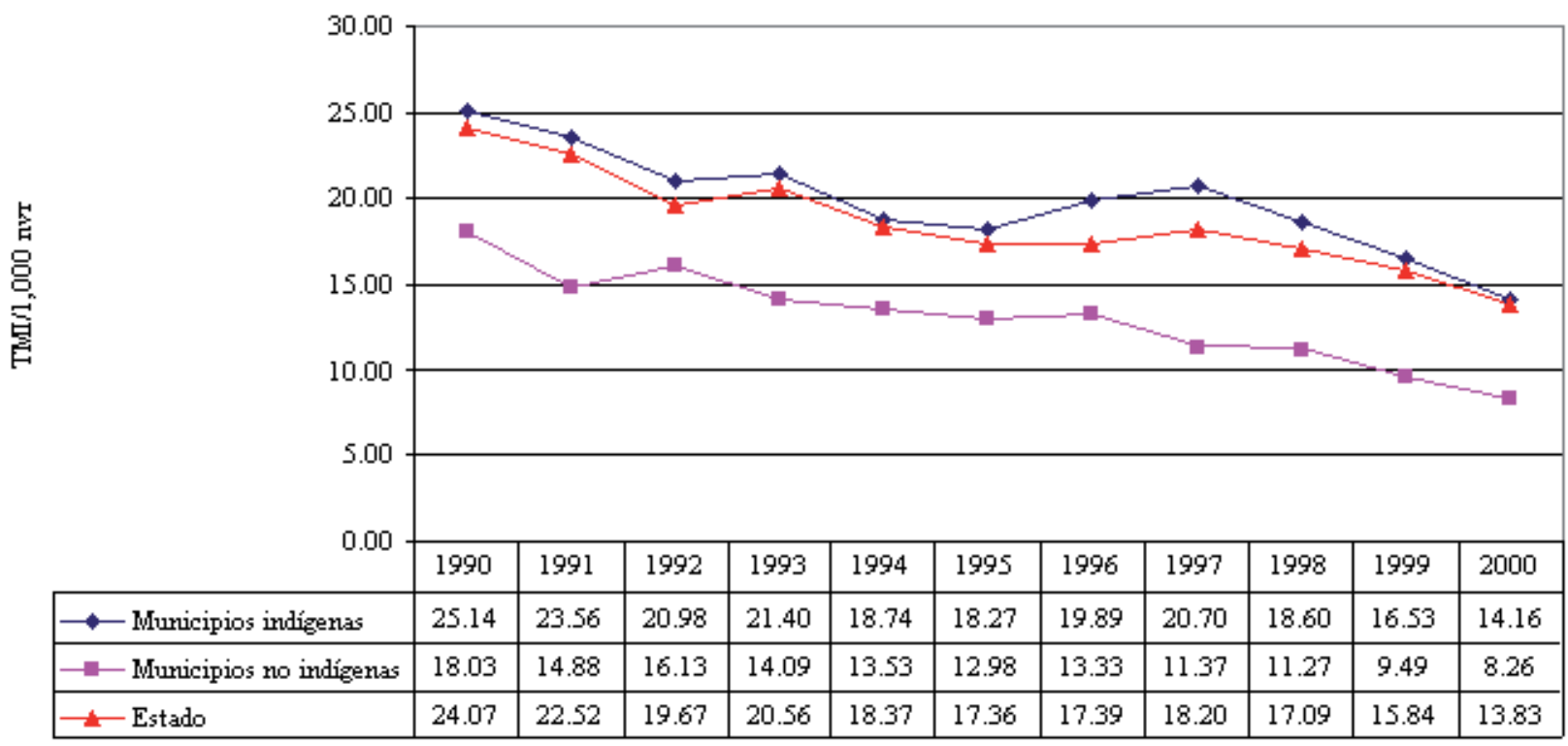

Fuente INEGI (Sistema municipal de Bases de Datos), 2003

*Municipio indígena: aquel en el que más del $40 \%$ de su póblación de 5 años y más habla lengua indígena (SSA-INI, 1992).

Figura 1.- Evolución de las tasas de mortalidad infantil (TMI) en Yucatán, México en municipios indígenas y no indígenas* 1990-200.

por encima de las TMI nacionales. La figura 1 muestra la evolución de las TMI de 1990 a 2000 para el estado, los municipios indígenas y los no indígenas. En todos los casos disminuyeron las TMI, aunque las de municipios indígenas están muy por arriba de las no indígenas y cercanas a las estatales. Además, la proporción de municipios indígenas con grado alto de mortalidad infantil se incrementó de $44.8 \%$ en 1990 a 50.7\% en 2000 . En ambos años, la mayoría de los municipios con grado alto de mortalidad infantil formaron una franja que se extendió del sur al oriente del estado.

Entre 1990 y 2000 el número de unidades médicas de primer nivel se incrementó en el estado, de 197 a 319 y, específicamente, en los municipios indígenas pasaron de 152 a 228. El número de unidades de segundo nivel disminuyó de 18 a 14 en el primer caso y de 10 a 5 en el segundo. Estas unidades se localizan en los municipios de Tizimín, Valladolid, Oxkutzcab, Maxcanú y Acanceh. Las unidades de tercer nivel aumentaron de 2 a 4 , pero éstas se concentraron en Mérida. En 1990 el 11.5\% de los municipios no contaba con ningún tipo de servicio médico, para el 2000 todos los municipios disponían de alguno.

Para el 2000, todos los municipios del estado contaron con médico. De 1990 a 2000 aumentó el número de médicos/1,000 habitantes a nivel estatal, pasando de 1.22 a 1.61. En cambio en los municipios indígenas, el aumento fue de menor magnitud pasó de 0.54 en 1990 a 0.78 en 2000. En ambos años predominaron los municipios que tuvieron menos de 1 médico/1,000 habitantes; sin embargo, la proporción de los mismos pasó de 75.9\% en 1990 a 73.4\% en 2000 .

\section{Análisis cartográficos relacionales.}

- Municipios indígenas que contaban con unidades médicas de primer nivel y presentaron grado alto de mortalidad infantil. En 1990, de los municipios indígenas que contaban con unidad médica de primer nivel, el 35\% presentó grado

Vol. 17/No. 2/Abril-Junio, 2006 


\section{RM Méndez-González, MD Cervera-Montejano.}

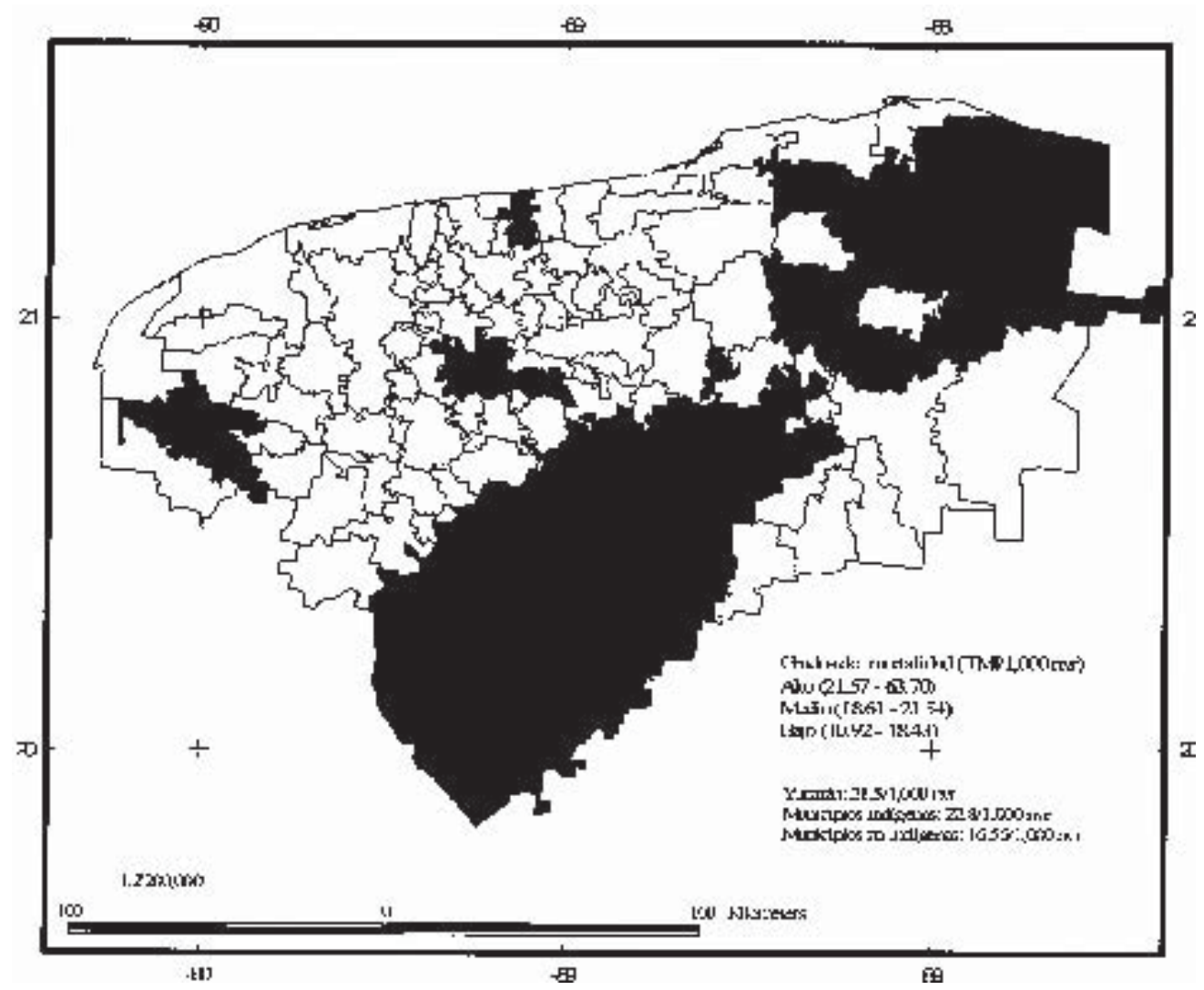

Fuente: INEGI, 1992, 2001, 2003

Mapa 1.- Municipios indígenas que contaban con unidades médicas de primer nivel y presentaron grado alto de mortalidad infantil en 1990, Yucatán, México.

alto de mortalidad infantil. En 2000 se incrementó a $47 \%$ la frecuencia de municipios que presentaron esta asociación. Los mapas 1 y 2 muestran los resultados de esta relación para 1990 y 2000, respectivamente. En ambos años se observa un conglomerado de municipios localizados en el sur y oriente del estado.

- Municipios indígenas que tuvieron grado alto de mortalidad infantil y menos de 1 médico/ 1,000 habitantes. De 1990 a 2000, la proporción de municipios indígenas con grado alto de mortalidad infantil y menos de 1 médico/1,000 habitantes aumentó de 35\% a 37\%. En los mapas 3 y 4 se despliega la distribución territorial que tuvo esta asociación de variables. En los dos años, la mayoría de estos municipios se localizaron en el sur y en el oriente del estado.

\section{DISCUSIÓN.}

Los resultados muestran que de manera similar a otras poblaciones indígenas del país, como las de Oaxaca, Chiapas, y Quintana Roo, las de Yucatán tienen un atraso importante en salud (46) ocasionado por tasas elevadas de mortalidad infantil, cuyas causas son debidas principalmente a enfermedades que pueden prevenirse, como las afecciones originadas en el período perinatal, las enfermedades infecciosas intestinales y las neumonías. Se ha reportado que la tasa de mortalidad infantil en estas poblaciones indígenas es dos veces superior a la nacional (1).

Entre 1990 y 2000, las TMI de los municipios indígenas de Yucatán se mantuvieron por encima de los municipios no indígenas y se incrementó el número de municipios indígenas que presentaron grado alto de mortalidad infantil. El peso de las TMI en estos municipios se refleja en las TMI

\section{Revista Biomédica}


Mortalidad infantil y servicios de salud en Yucatán, México, 1990-2000.

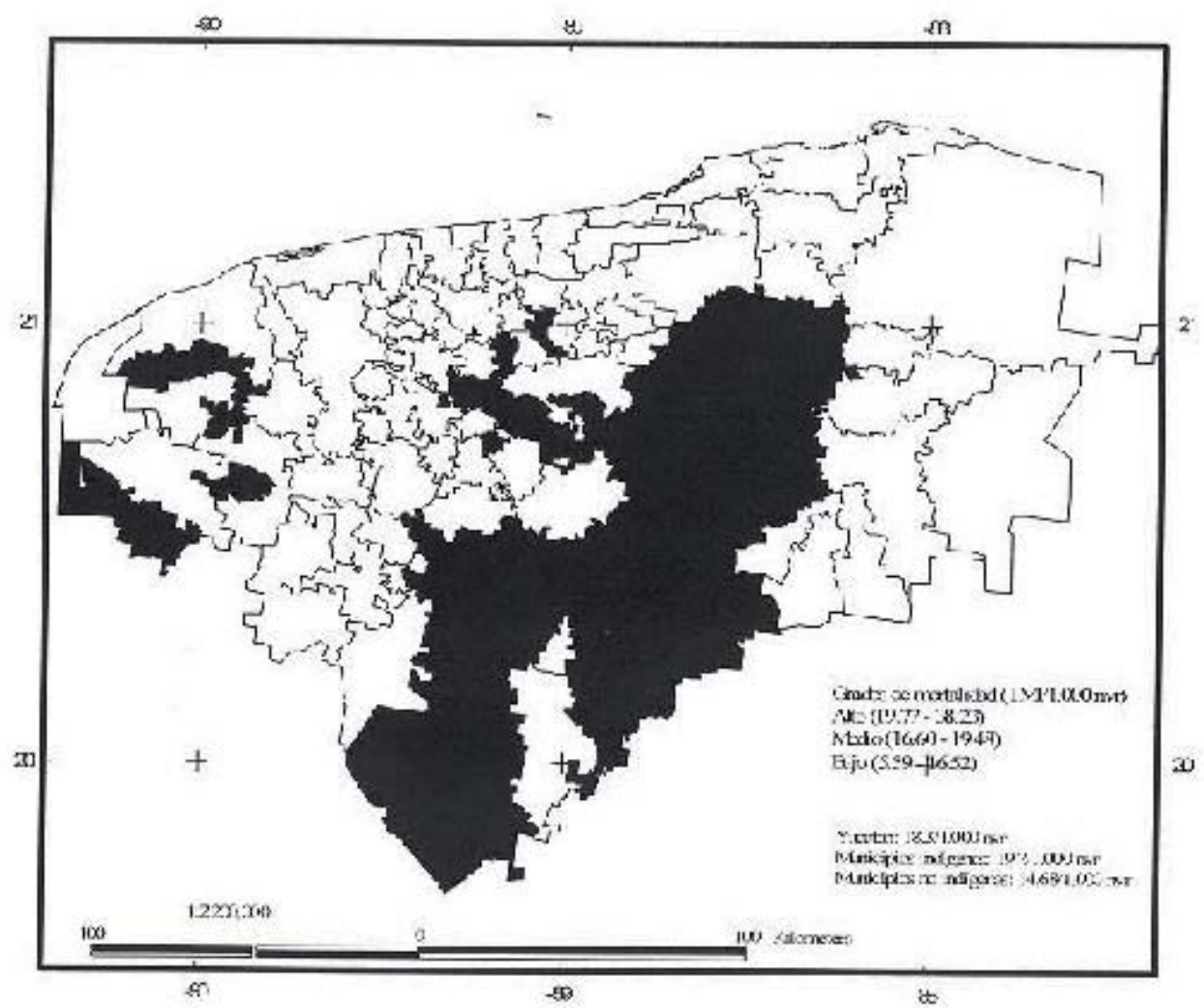

Fuente: INEGI, 1992, 2001, 2003

Mapa 2.- Municipios indígenas que contaban con unidades médicas de primer nivel y presentaron grado alto de mortalidad infantil en 2000, Yucatán, México.

estatales, las cuales son muy similares.

Quedó de manifiesto que las cifras estatales ocultan la situación de mortalidad infantil que ocurre en los municipios. En 1990, la media estatal de mortalidad infantil fue de 21.5/1,000 nvr, pero el rango de TMI fue muy amplio, con un valor mínimo de 10.9/1,000 nvr y uno máximo de 63.8/1,000 nvr; en 2000, la media estatal fue de 18.3/1,000 nvr, con valores mínimo y máximo de 5.6/1,000 nvr y 38.2/1,000 nvr, respectivamente.

De 1990 a 2000 aumentó el número de unidades médicas de primer nivel pero diminuyeron las de segundo nivel. Este descenso se debió a la transformación de éstas a unidades de primer nivel, aunque la construcción permaneció de segundo nivel, el servicio otorgado -recursos humanos y materiales- pasaron a ser de primer nivel. Se advirtió que las poblaciones indígenas que habitan en el centro del estado, se encuentran muy alejadas de las unidades médicas de segundo nivel. El número de médicos/1,000 habitantes se mantuvo bajo y no mejoró con respecto a la proporción que correspondió al estado. En 1990, el número de médicos/1,000 habitantes en las comunidades indígenas representó el $44.3 \%$ del reportado a nivel estatal (0.54 contra 1.22) y en 2000 representó el 48.5\% (0.78 contra 1.61).

Los resultados de la relación espacial mostraron que de 1990 a 2000 se incrementó la proporción de municipios indígenas que contaban con unidades médicas de primer nivel de atención y tuvieron grado alto de mortalidad infantil (de $35 \%$ a $47 \%)$. Asimismo, aumentó la frecuencia de los que tenían grado alto de mortalidad infantil y menos de un médico/1,000 habitantes (de 35 a 37\%). Si bien se incrementó la infraestructura de servicios de salud, el número de médicos para brindar la atención requerida parece insuficiente. La 


\section{RM Méndez-González, MD Cervera-Montejano.}

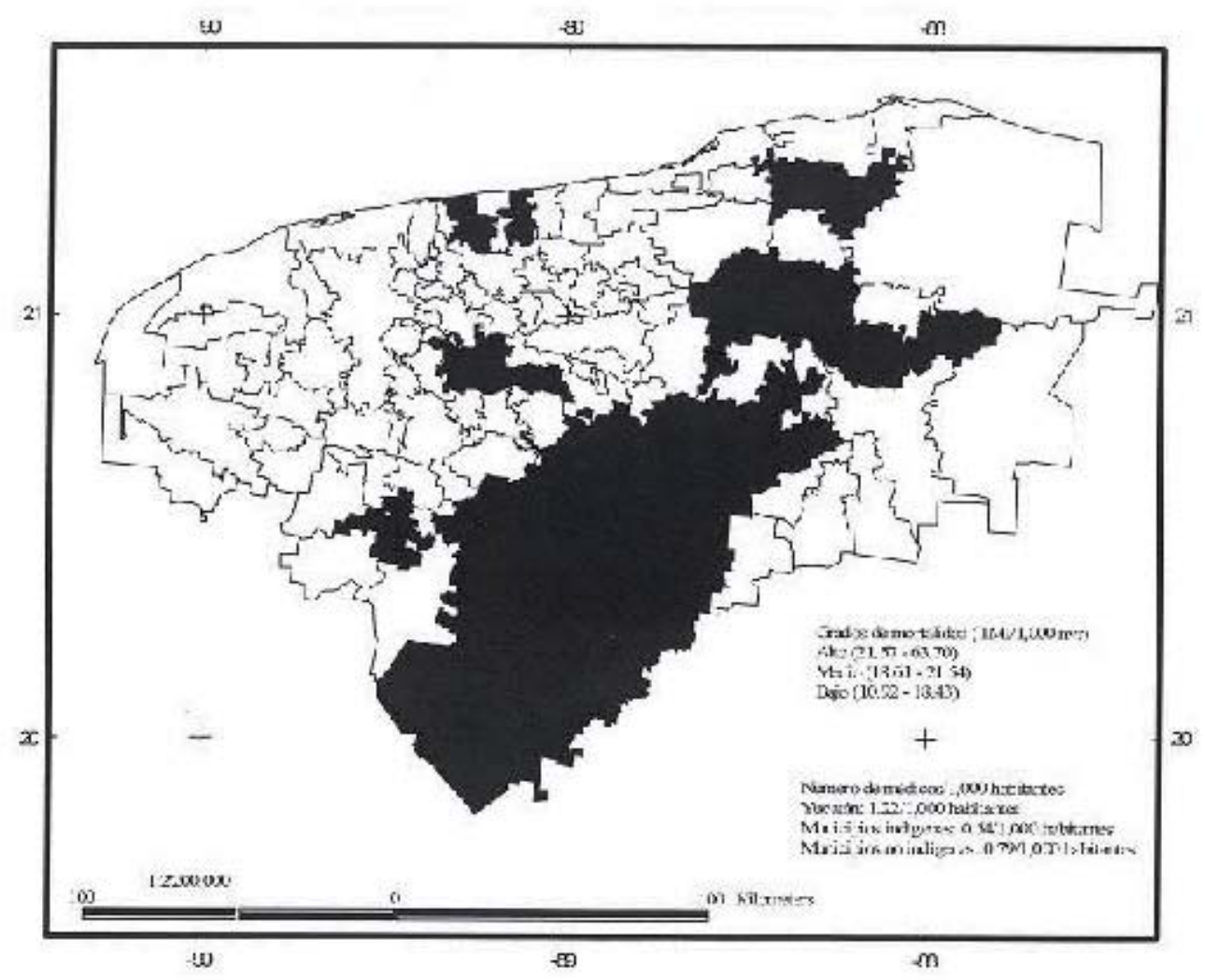

Fuente: INEGI, 1992, 2001, 2003

Mapa 3.- Municipios indígenas que tuvieron grado alto de mortalidad infantil y menos de 1 médico/1,000 habitantes en 1990, Yucatán, México.

disponibilidad de servicios de salud por sí misma no involucra su utilización ni su relación directa con la disminución de las tasas de mortalidad infantil. Estos hallazgos coinciden con el planteamiento de que la mortalidad infantil se relaciona con varios factores, además de la desigualdad en el ingreso económico, con la disponibilidad y el acceso de los servicios básicos de salud (7-13).

Por ello, sería necesario investigar si se trata de un problema de disponibilidad o de acceso a los servicios de salud e identificar cuáles son las barreras que interfieren con el uso de los servicios, porque podría ser que en la mayoría de los casos se tratara de un problema de acceso más que de disponibilidad.

Una de las limitaciones del estudio fue el uso de promedios de tasas de mortalidad infantil de los municipios para los períodos 1990-1994 y 1995-2000, ya que éstos pueden ocultar lo que sucede cada año en el municipio. Sin embargo, en geografía de la salud, se recomienda utilizarlos en los casos en que se estudian tendencias espaciotemporales, considerando que un solo valor no refleja el comportamiento de la mortalidad $(19,22)$.

El análisis espacio-temporal fue fundamental porque permitió identificar los cambios ocurridos en la década y distinguir que las zonas sur y oriente del estado fueron las más desfavorecidas $\mathrm{y}$, por lo tanto, las que requieren de atención prioritaria. El Sistema de Información Geográfica fue una herramienta importante que facilitó la visualización y el análisis de datos, la identificación de relaciones espaciales entre variables y de los cambios que ocurrieron en la década 19902000. Los SIG constituyen una herramienta analítica muy importante en salud pública y epidemiología porque permiten la descripción

\section{Revista Biomédica}




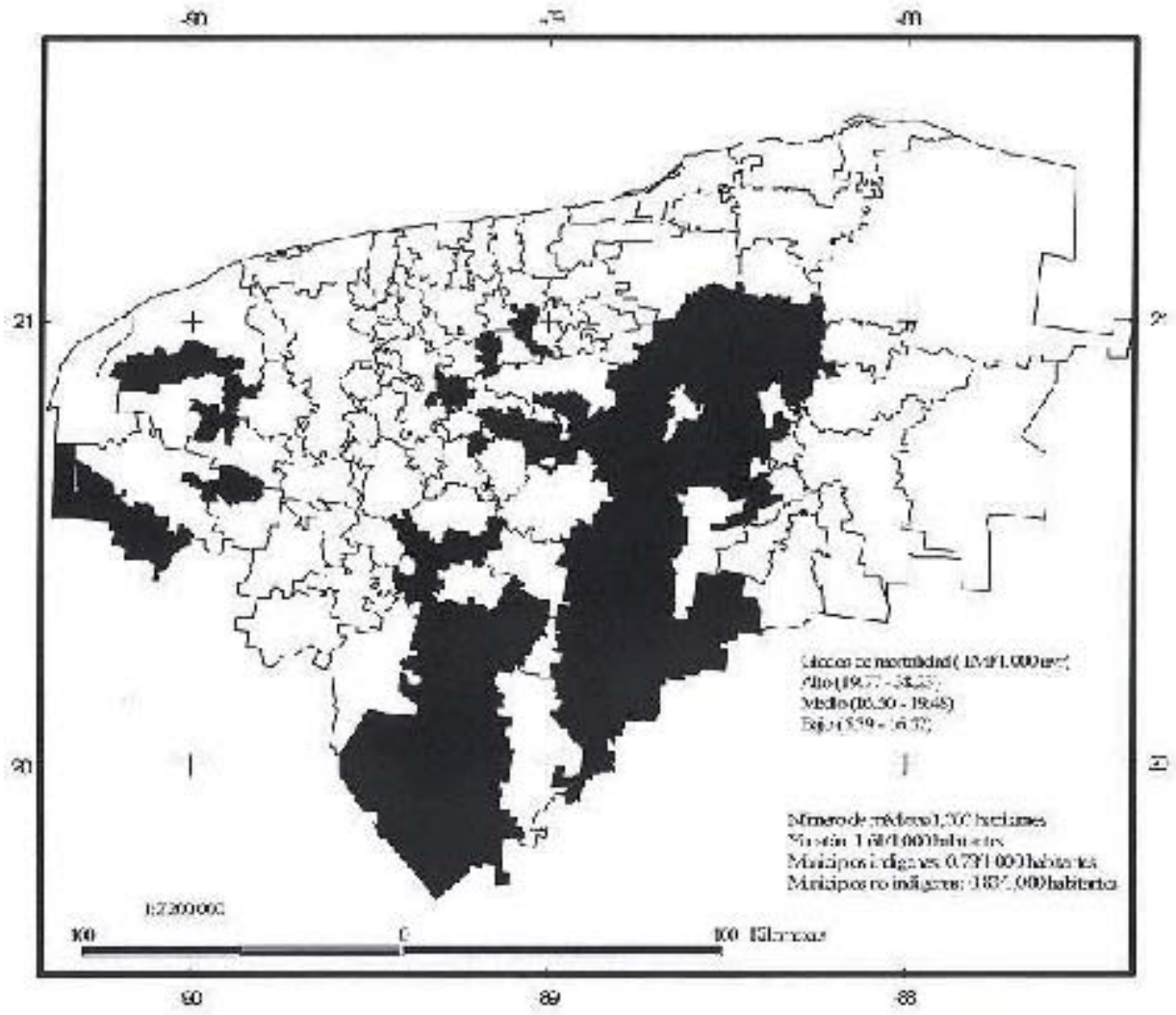

Fuente: INEGI, 1992, 2001, 2003

Mapa 4.- Municipios indígenas que tuvieron grado alto de mortalidad infantil y menos de 1 médico/1,000 habitantes en 2000, Yucatán, México.

espacial de la situación de un evento de salud en un área geográfica. A través de los SIG se pueden observar y predecir tendencias e identificar interrelaciones entre factores condicionantes específicos y apoyar la toma de decisiones sobre intervenciones apropiadas del sector salud (23). El análisis espacial y el uso de los SIG en el área de la salud ha sido muy variado. Por ejemplo, se han utilizado para determinar la distribución geográfica de la morbilidad, mapear poblaciones en riesgo, describir espacialmente un evento de salud, analizar espacialmente la mortalidad, etc. La Organización Panamericana de la Salud ha promovido el uso y desarrollo de los SIG en la región de las Américas, por la importancia que tiene su aplicación en epidemiología y salud pública (23).

\section{REFERENCIAS.}

1.- OPS. Organización Panamericana de la Salud. La salud en las Américas. Publicación científica 587, 2002; 1:1-22.

2.- WHO. World Health Organization. Equity in health and health care: a WHO initiative. Ginebra; 1996.

3.- OPS. Organización Panamericana de la Salud. Resolution $\mathrm{V}$ of the Meeting on Indigenous Peoples and Health. Winnipeg, Canadá. Document CD37/20; 1993.

4.- Torres JL, Villoro R, Ramírez T, Zurita B, Hernández P, Lozano R. La salud de la población indígena en México. Caleidoscopio de la Salud México FUNSALUD; 2003: 41-54.

5.- Lozano R, Zurita B, Franco F, Ramírez T, Hernández P, Torres JL. México: marginality need and resource allocation at the county level. En: Evans T. Whitehead M. Diderichsen F, Bhuiya A. Wirth M (eds) Challenging inequities in health: form ethics to action. Nueva York. The Rockefeller Foundation. Oxford University Press; 2001. p. 227-295 


\section{RM Méndez-González, MD Cervera-Montejano.}

6.- SSA. Secretaría de Salud. Programa Nacional de Salud 2001-2006. La democratización de la salud en México. Hacia un sistema universal de salud. Secretaría de Salud, México; 2001

7.- Behm, H. Socioeconomic determinants of mortality in Latin America. In: Proceedings of the Meeting on Socioeconomic Determinants and Consequences of Mortality, Geneva, World Health Organization 1979; 140165.

8.- Solimano G, Chapin G. The impact of socioeconomic development and ecological change on health and nutrition in Latin America. Cornell International Nutrition Monograph Series Ithaca, NY. Cornell University 1981;(9).

9.- Mosley W, Chen LC. An analytical framework for the study of child survival in developing countries. Population and Development Review; 1984; 10 (Suppl 10): 25-45.

10.- Masuy-Stoobant G, Gourbin C. Infant Health and mortality indicators. European Journal of Population; 1995; 11: 63-84.

11.- OPS. Organización Panamericana de la Salud, La salud de las Américas. Riesgos de morir y desigualdades en el ingreso. Boletín Epidemiológico 1999; 20(4):7-10.

12.- OPS. Organización Panamericana de la Salud. Desigualdades en la mortalidad infantil en la región de las Américas: elementos básicos para su análisis. Boletín Epidemiológico 2001; 22(2): 4-7.

13.- SSA. Secretaría de Salud. Estadísticas vitales. Series históricas. 2001 Disponible en: http://www.ssa.gob.mx.

14.- INEGI. Instituto Nacional de Estadística, Geografía e Informática. La población indígena en México; 2004.

15.- INEGI. Instituto Nacional de Estadística, Geografía e Informática, XI Censo General de Población y Vivienda 1990, Tabulados básicos y por entidad federativa, Bases de datos y tabulados de la muestra censal, México; 1990.

16.- INEGI. Instituto Nacional de Estadística, Geografía e Informática, XII Censo General de Población y Vivienda 2000, Tabulados básicos y por entidad federativa, Bases de datos y tabulados de la muestra censal, México; 2000.

17.- SSA-INI. Secretaría de Salud-Instituto Nacional Indigenista. La salud de los pueblos indígenas en México. 1992.

\section{Revista Biomédica}

18.- INEGI. Instituto Nacional de Estadística, Geografía e Informática. Sistema Municipal de Bases de Datos (SIMBAD); 2003 Disponible en: http://www.inegi.gob.mx

19.- Meade M, and Earickson RJ. Scale, spatial analysis, and geographic visualization. Medical Geography. The Guilford Press, New York-London; 2000. p. 438-484.

20.- INEGI. Instituto Nacional de Estadística, Geografía e Informática. Anuario Estadístico del Estado de Yucatán, México; 1992.

21.- INEGI. Instituto Nacional de Estadística, Geografía e Informática. Anuario Estadístico del Estado de Yucatán, México; 2001.

22.- Gatrell, AC. Geographies of Health: An Introduction. Blackwell Publishers. Oxford, UK, 2002.

23.- OPS. Organización Panamericana de la Salud. Sistemas de Información Geográfica en Salud. Conceptos básicos. Washington, D.C. 2002. 